\title{
Design and analysis of an OFDM-based orthogonal multilevel code-shifted differential chaos shift keying
}

\author{
Fadhil S. Hasan, Hayder F. Fahad \\ Electrical Engineering Department, Collage of Engineering, Al Mustansiriyah University, Iraq
}

\begin{tabular}{|c|c|}
\hline Article Info & ABSTRACT \\
\hline Article history: & In this paper, a new non-coherent chaos-based communication that combined \\
\hline Received Nov 28, 2019 & orthogonal multilevel code-shifted differential chaos shift keying with \\
\hline Revised Mar 20, 2020 & $\begin{array}{l}\text { modulation system. New orthogonal chaotic signal sets are generated by } \\
\text { mats }\end{array}$ \\
\hline Accepted Jun 5, 2020 & $\begin{array}{l}\text { hybrid gram-schmidt algorithm and walsh code function and used it to carry } \\
\text { high data rate in the same time slot. The bandwidth efficiency of the OFDM- }\end{array}$ \\
\hline Keywords: & $\begin{array}{l}\text { OMCS-DCSK system is comparing with conventional system. Also, the BER } \\
\text { analytic expressions are derived under (AWGN) and multipath Rayleigh }\end{array}$ \\
\hline $\begin{array}{l}\text { Gram-Schmidt algorithm } \\
\text { High data rate }\end{array}$ & $\begin{array}{l}\text { fading channels and comparing with the simulation results. Furthermore, the } \\
\text { BER performance is compared with OFDM-DCSK to found that the } \\
\text { proposed system has the best BER performance with high data rate feature. }\end{array}$ \\
\hline
\end{tabular}

Copyright $@ 2020$ Institute of Advanced Engineering and Science. All rights reserved.

\section{Corresponding Author:}

Fadhil S. Hasan,

Department Electrical Engineering,

Mustansiriyah University, Baghdad, Iraq.

Email: fadel_sahib@uomustansiriyah.edu.iq

\section{INTRODUCTION}

Through the last years have begun to be utilized chaos signals in digital communication system, to benefit from the characteristics of chaotic signals, for instance, wideband spectrum, perfect randomness conduct [1], simple to generate a sequence [2], and good correlation properties [3] make them it appropriate utilized for digital communication and spread spectrum systems. Besides, it has a robust immunity in multipath fading channels [4], the weakened probability to intercept and jamming [5], higher sensitivity to initial conditions [6] and chaotic systems mimic chaotic systems since both systems are extremely unstable. Chaotic systems have been pseudorandom used in cryptography [7]. The chaos-based digital communication system can be classified into coherent and non-coherent schemes. In the first scheme, such as chaos shift keying (CSK) system, the synchronization between transmitter and receiver and the dependence of the channel state information must be satisfied, which difficulty practically applied [2]. On the other side, the non-coherent scheme such as the differential chaos shift keying (DCSK) is more popular use in chaos-based digital communication systems without requiring the synchronization and information of the channel state [4].

The DCSK system utilizes two-time slots to each transmitted bit [8], the first send the reference chaotic and the second data-bearing. DCSK system solved the problem of the synchronization at the expense of reducing the data rate, weakened security, and high energy consumption [3,9]. Therefore, in recent years, many proposals have been made to enhance the DCSK system drawbacks [9-28]. One of the most important limitations of DCSK system is the low data rate. In [10] Quadrature chaos shift keying (QCSK) was proposed to double the data rate at the same bandwidth to DCSK by helping Hilbert transform filter. In [11] M-ary 
DCSK system was proposed with a higher data rate from the QCSK system and gave better BER performance from the M-ary PSK-DCSK system (MPSK-DCSK) [12].

In [13-20], orthogonal chaotic vector shift keying (OCVSK), Multilevel Code-Shifted DCSK (CS-DCSK), high-efficiency DCSK (HEDCSK), Multi-Carrier DCSK (MC-DCSK) system, high data rate code shifted DCSK (HCS-DCSK), reference modulate DCSK (RM-DCSK), multilevel code-shifted DCSK (MCS-DCSK), and orthogonal multilevel DCSK (OM-DCSK) respectively utilized to enhance the information rate. Another approach to improve the data rate to decrease the length of the reference phase-separated DCSK (PS-DCS) [21], improved DCSK (I-DCSK) [22], short reference DCSK (SR-DCSK) [23], commutation code index DCSK (CCI-DCSK) [24]. It provides a very high data rate and is used in the wireless communication network. OFDM is a very common technique [25]. In other studies, to enhance the bandwidth efficiency by hybrid Multicarrier modulation and orthogonal frequency division multiplexing (OFDM) with DCSK such as (OFDM-DCSK) [26], OFDM based Short Reference-QCSK (OFDM-SRQCSK) [27], OFDM based OCVSK (OFDM-OCVSK) [9] and a multicarrier DCSK (MC-DCSK) system [28, 29].

Furthermore, the last studies which hybrid the index modulation (IM) [30] with DCSK system promised the efficient way to increase the data rate such as Coded Index Modulation (CIM) [31], Carrier Index DCSK (CI-DCSK) [32], CIM-CS-DCSK [33], and CIM-OMDCSK [34]. In this paper, a new solution of high data rate system is proposed which hybrid an OFDM system, OM-DCSK and Grams Schmidt process named OFDM based Orthogonal Multilevel Code Shifted DCSK (OFDM-OMCS-DCSK). Orthogonal Multilevel Code Shifted DCSK (OMCS-DCSK) used Gram-Schmidt algorithm as in [16] and Walsh function as in [22] to generate new orthogonal set that carry higher data in the same time slot comparing with OCVSK and OM-DCSK. The proposed orthogonal sets are capable of robust against the interference at the receiver side, increased transmitted bits to $(\mathrm{M} \times \mathrm{N})$ bits and improve the BER performance. The BER analytic of the proposed system over AWGN and multipath Rayleigh fading channels are derived and compared with simulation results and the conventional OFDM-DCSK system.

The rest of the paper is organized as follows: Section II presents OFDM-OMCS-DCSK system transmitter and receiver structures. Section III derives Bandwidth efficiency and the analytical BER at AWGN channel and multipath Rayleigh channel. Section IV presents the simulation results. Finally, Section V contains the conclusion.

\section{OFDM-OMCS-DCSK SYSTEM}

In this section, the transmitter and receiver schemes of OFDM-OMCS-DCSK system are presented and demonstrated in details.

\subsection{Transmitter}

Figure 1 shows the block diagram of an OFDM-OMCS-DCSK transmitter. In the first step, M $\times \mathrm{N}$ orthogonal vectors are generated using the repeated orthogonal chaotic generator (ROCG) and Walsh function. ROCG is duplicated in Figure 2. The source of $N$ chaotic sequence, $x_{\mathrm{j}}^{(n)}, \mathrm{j}=0, \ldots, N-1$ are produced, where $n \in[0, R)$ and $R$ is the spread factor length. The chaotic sequence is produced using Chebyshev second-order equation Multiprocessor Function (CPF) with variance $=1$ and mean $=0$ as [27].

$$
x_{n+1}=1-2 x_{n}^{2}
$$

Then Gram-Schmidt process is suggested to generate $N$ orthogonal chaotic signals from non-orthogonal sequences, $x_{g j}^{(n)}, j \in[0, N)$ and $n \in[0, R)$. Then, ROCG is used to produces the duplicated sequences, $x_{g}^{(n)}(j, i), n \in[0, R)$, from the $\mathrm{j}$-th chaotic sequence, $x_{g j}^{(n)}$ by duplicating $(M-1)$, where $j \in[0, N), i \in[0, M)$. Then using Walsh function and the resulting of ROCG to produce $M \times N$ orthogonal chaotic sequences according to

$$
x_{b}^{(q)}(\mathrm{j}, \mathrm{i})=\sum_{k=0}^{M-1} W_{i k} x_{g j}^{(n)} \operatorname{rect}(n-k R), \underset{j \in[0, M)}{i \in[0, N),} \quad \begin{gathered}
q \in[0, M \times R) \\
n \in[0, R)
\end{gathered}
$$

where $W_{i k}$ is the $i$-th row and $k$-th column of Walsh function that is produced by Hadamard matrix according

$$
W_{2^{\varphi}}=\left[\begin{array}{cc}
W_{2^{\varphi-1}} & W_{2^{\varphi-1}} \\
W_{2^{\varphi-1}} & -W_{2^{\varphi-1}}
\end{array}\right], W_{2^{0}}=[1] \text { for } \varphi=1,2, . .
$$


and $\operatorname{rect}(n-k R)$ is a discrete rectangular function that is written as

$$
\operatorname{rect}(n-k R)= \begin{cases}1 & k R \leq n<(k+1) R \\ 0 & \text { otherwise }\end{cases}
$$

These $M \times N$ orthogonal sets are capable to carry $M \times N$ bits on the same time slot. In the second step, each serial bit $b_{m}$ is mapped into the symbol $a_{m}=\{1,-1\}$. The serial m-th symbol is then converted into $\mathrm{P} \times \mathrm{N} \times \mathrm{M}$ parallel symbols $\mathrm{d}_{\mathrm{m}, \mathrm{k}}$ with $\mathrm{k}=0, \ldots . \mathrm{P} \times \mathrm{N} \times \mathrm{M}-1$. Then, the resulting symbols are divided into $\mathrm{P}$ groups, each of $\mathrm{N} \times \mathrm{M}$ symbols. Each $p$-th group is modulated using what is named OMCS-DCSK in which they are summing in the same time slot to produce the $p$-th signal $S_{m}(p, q)$ as

$$
S_{m}(p, q)=\frac{1}{\sqrt{M N}} \sum_{j=0}^{N-1} \sum_{i=0}^{M-1} a_{m,(i+j M+(p-1) M N)} x_{b}^{(q)}(\mathrm{j}, \mathrm{i}) \quad, 0 \leq q<M R, 1 \leq p \leq P
$$

The first $S_{m}(0, n)$ is occupied by all the multiplexed reference signals $\mathrm{X}_{\text {ref }}=\left[\begin{array}{llll}x_{g 0}^{(n)} & x_{g 1}^{(n)} \ldots . & x_{g(N-1)}^{(n)}\end{array}\right]$, where $\mathrm{X}_{\text {ref }} \in \mathbb{R}_{N R \times 1}$. Here $\mathrm{M}=\mathrm{N}$ for all system. Without loss of the generality, if $m$ is dropped from the equation, then the discrete signal before IFFT is expressed as

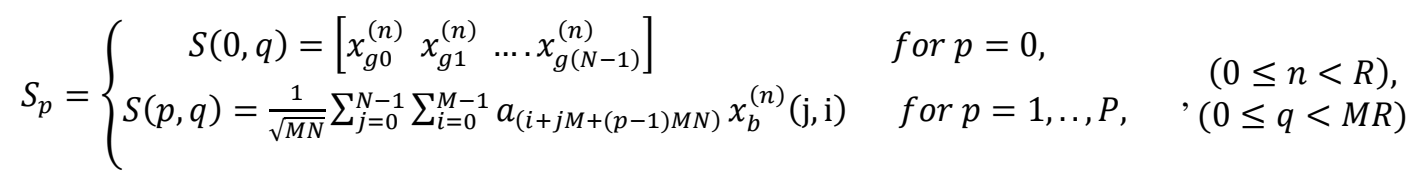

In the last step of transmitted side, each $q$-th column of the $S(p, q)$ matrix is passed through the inverse FFT to generate OFDM signal with subcarrier number, $N_{F F T}=P+1$ as minimum. The baseband discrete OFDM-OMCS-DCSK modulated signal with cyclic extended guard interval is written as

$$
s(v, q)=\frac{1}{\sqrt{N_{F F T}}} \sum_{p=0}^{N_{F F T}-1} S(p, q) e^{j 2 \pi \frac{p v}{N_{F F T}},} \quad \begin{gathered}
v=-N_{g}, \ldots, N_{F F T}-1 \\
(0 \leq q<M R)
\end{gathered}
$$

where $N_{g}$ is the guard interval, $N_{g}=\left[0.25 N_{F F T}\right\rceil$, and $\lceil$.$\rceil is the integer function. The frame structure of OFDM$ OMCS-DCSK signal is illustrated in Figure 3. The frame duration, $\mathrm{T}_{\text {OFDM-OMCS-DCSK }}=M(1+P) R \mathrm{~T}_{\mathrm{c}}$, where $\mathrm{T}_{\mathrm{c}}$ is the chip period. The energy bit of the transmitted signal, $E_{b}=\frac{(1+P) R}{M P} E\left[\left(x_{g j}^{n}\right)^{2}\right]$, where E[.] is the average value operator.

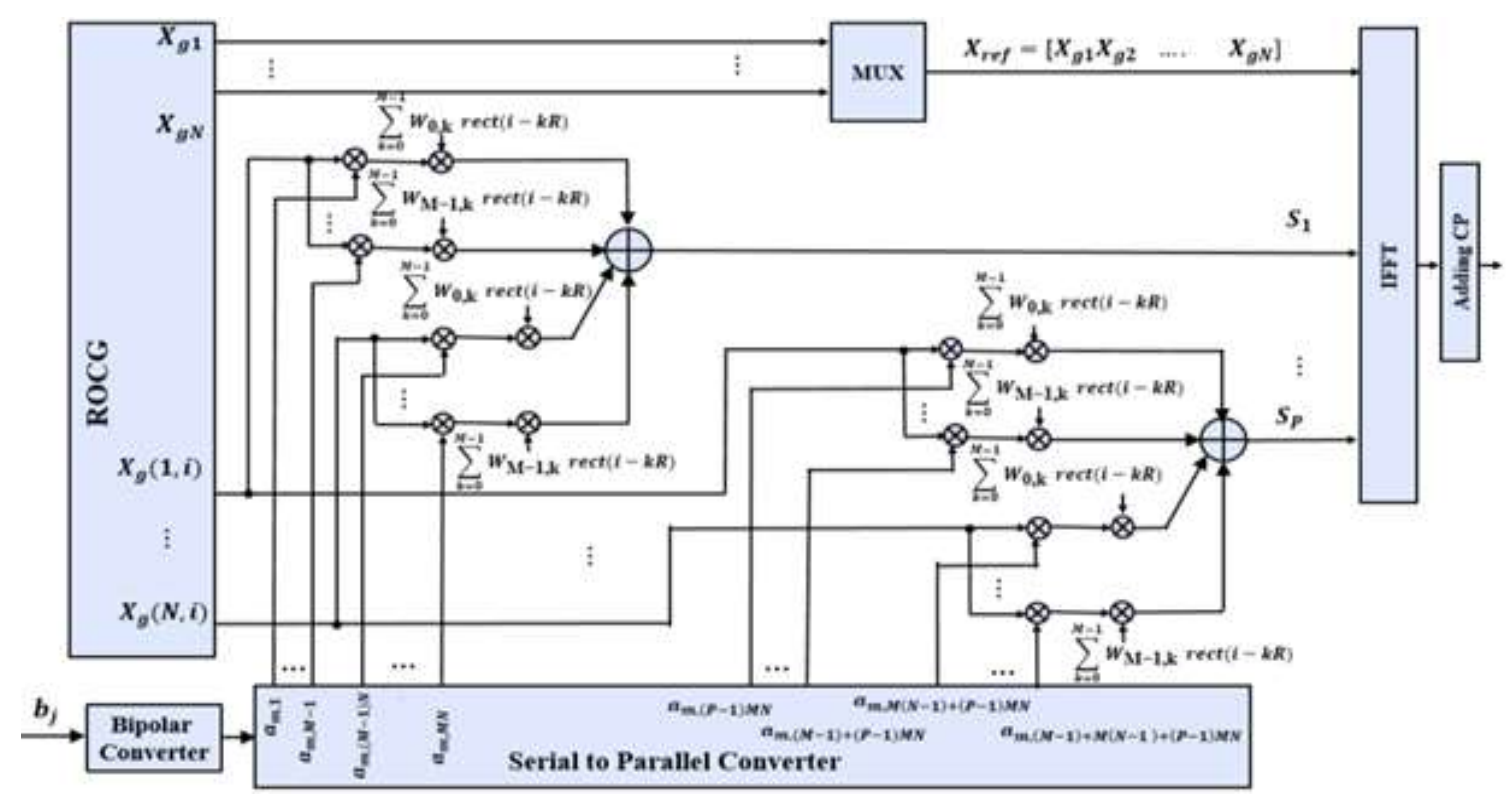

Figure 1. Structure of OFDM-OMCS-DCSK transmitter 


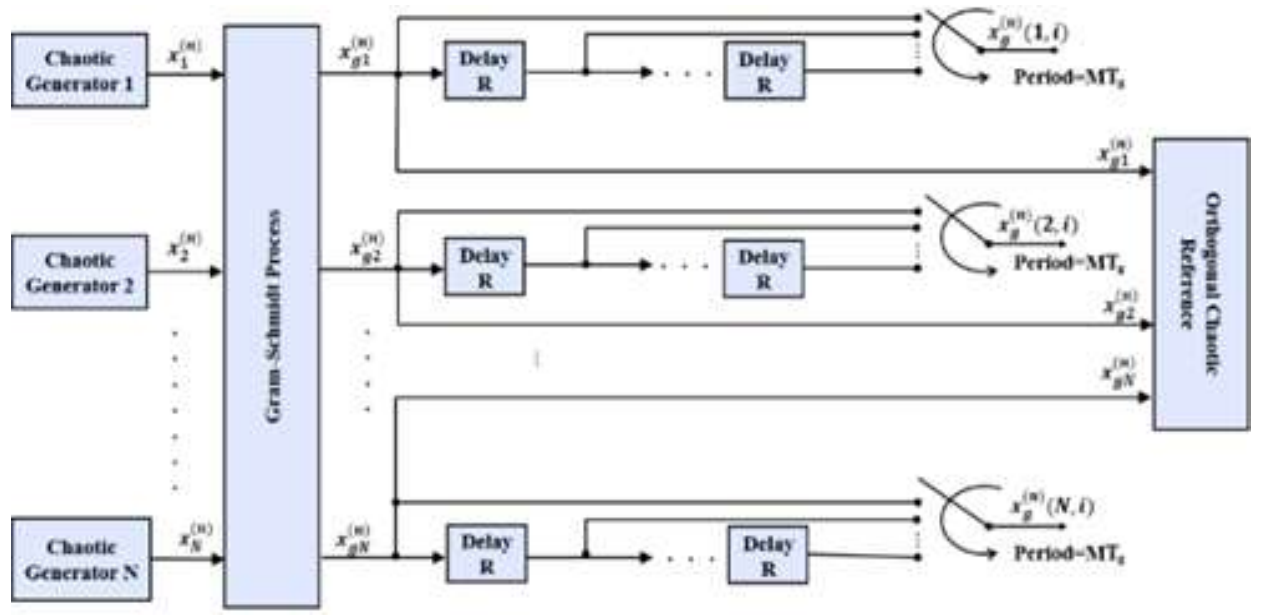

Figure 2. Structure of ROCG

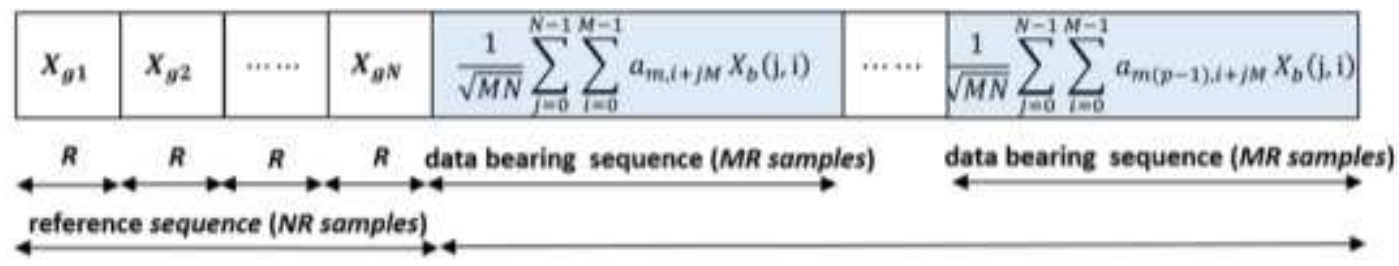

Figure 3. OFDM-OMCS-DCSK frame

\subsection{Receiver}

Figure 4 shows the block diagram of OFDM-OMCS-DCSK receiver. The first step is removing the cyclic prefix and taking an FFT function from the received complex signal $r(v, q)$ to perform the $p$-th demodulated complex signal $\mathrm{R}(\mathrm{p}, \mathrm{q})$ as

$$
R(p, q)=\frac{1}{\sqrt{N_{F F T}}} \sum_{v=0}^{N_{F F T}-1} r(v, q) e^{-j 2 \pi \frac{v p}{N_{F F T}}}, \quad \begin{gathered}
\quad=0, \ldots, N_{F F T}-1 \\
(0 \leq q<M R)
\end{gathered}
$$

From the sequence $R(0, q)$, the $i$-th received reference sequence, $x_{g j}^{(n)}+\xi_{\mathrm{n}+\mathrm{j} R}, \mathrm{j}=0, . ., \mathrm{N}-1$, $0 \leq n<R$, are extracted. Each received reference sequence is duplicated $M$ times using repeated orthogonal chaotic at receive (ROCR) as shown in Figure 5 to produce.

$$
y_{r j}^{(n+k R)}=\left[x_{g j}^{(n)}+\xi_{\mathrm{n}+\mathrm{j} R} \ldots \ldots x_{g j}^{(n)}+\xi_{\mathrm{n}+\mathrm{j} R}\right], n=0, . ., R-1, k=0, \ldots, M-1
$$

From (8) $M \times N$ orthogonal basis are produced by multiplying it with the Walsh code according to

$$
r_{b(n+k R)}(\mathrm{j}, \mathrm{i})=\sum_{k=0}^{M-1} W_{i k} x_{r}(n+k R)+\xi(n+k R) \quad \begin{aligned}
j & =0,1, \ldots, N-1 \\
i & =0,1, \ldots, M-1
\end{aligned}
$$

These reference chaotic basis can be used to extract all the $M \times N \times P$ bits. The $p$-th group of received data bearing sequence is written as

$$
\begin{aligned}
& y_{d}(p, n+k R)=\frac{1}{\sqrt{M N}} \sum_{j=0}^{N-1} \sum_{i=0}^{M-1} a_{i+j M+(p-1) M N} W_{i k} x_{r}(n+k R)+\xi(\mathrm{p}, n+M R+k R), \\
& 0 \leq k<M, 0 \leq n<R, 1 \leq p \leq P
\end{aligned}
$$

To recover the first bits in the 0 -th group, which are the $v$-th transmitted bits in the $u$-th subgroup, the received information carrying sequence, $y_{d}(0, n+k R)$ is multiplying with the sequence, $r_{b}^{(n+k R)}(u, v)$ 
and the energy value is found and compared to a zero threshold. The output of the $v$-th correlator in the $u$-th subgroup is written as

$$
Z_{u, v}=\sum_{k=0}^{M-1} \sum_{n=0}^{R-1} r_{b(n+k R)}(u, v) y_{d}(n+k R), 0 \leq u<N, 0 \leq v<M
$$

The $u$-th recovered bit in the $v$-th subgroup, $\hat{a}_{u+v M}$ is calculated from the decision value $D_{u+v M}$ by comparing the correlator output, $Z_{u, v}$ to a zero threshold value. After that the parallel to serial converter is used to get the stream recovered bits.

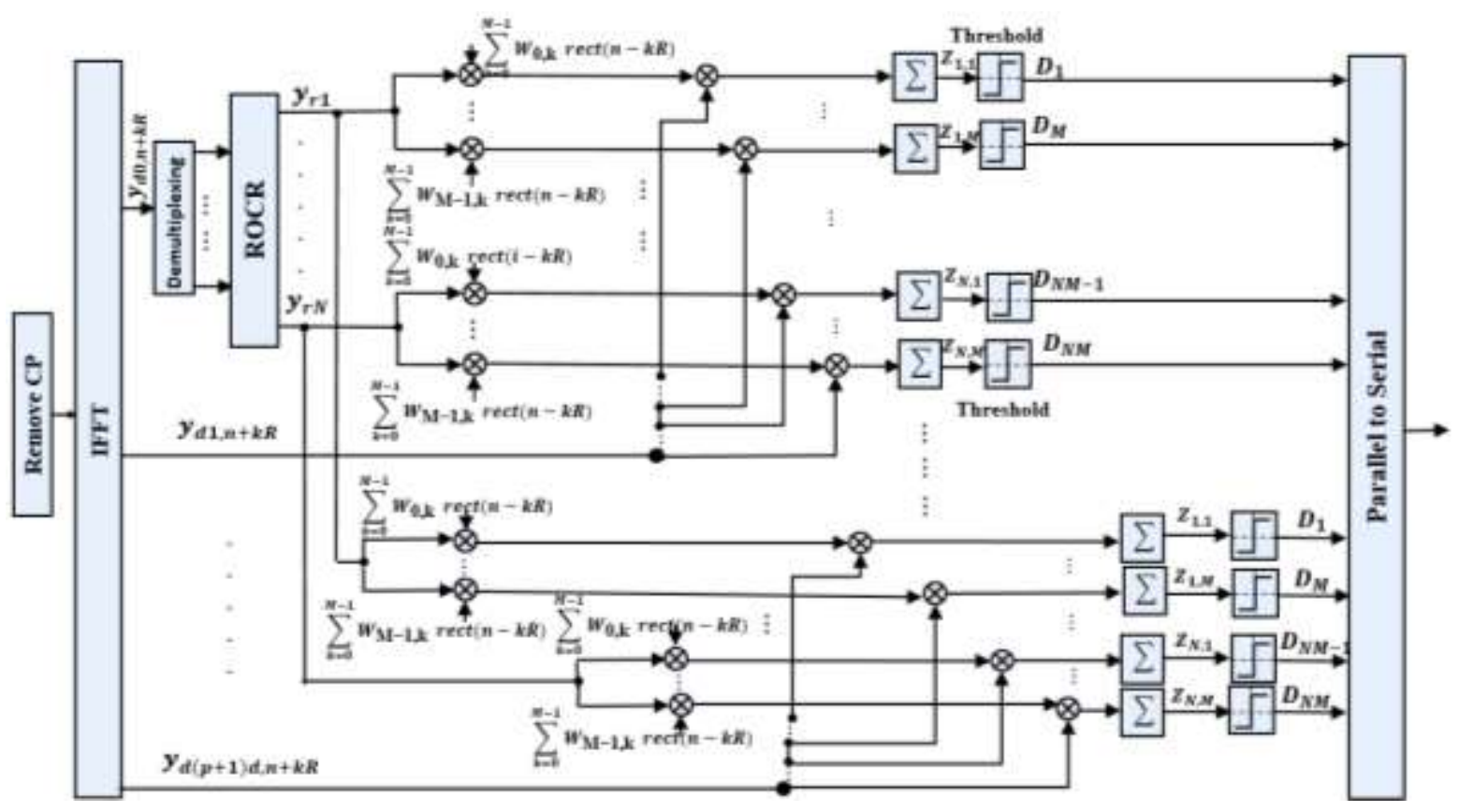

Figure 4. Block diagram of OFDM-OMCS-DCSK receiver

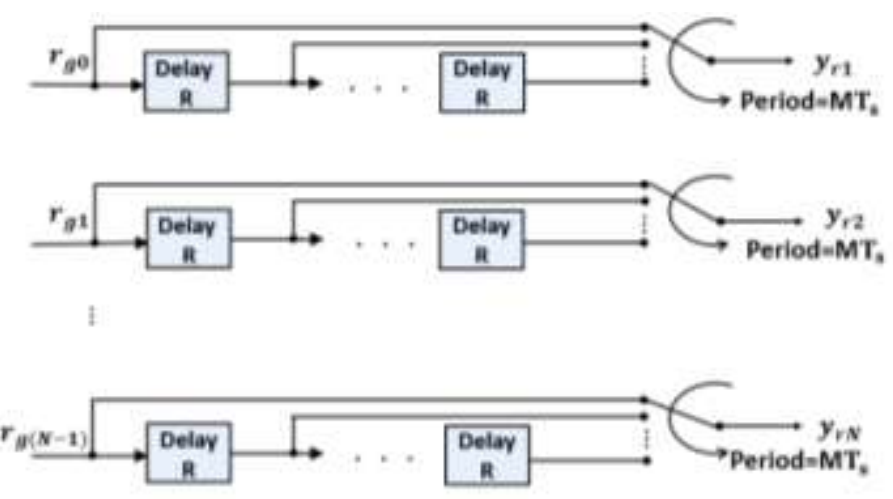

Figure 5. Structure of ROCR

\section{PERFORMANCE ANALYSIS}

In this section, the analytical BER of OFDM-OMCS-DCSK system is derived under multipath Rayleigh fading channel and AWGN.

\subsection{BER Analysis of OFDM -OMCS-DCSK}

\subsubsection{BER over an AWGN channel}

Since all group is the same bits streams independently and have the same error probability to recover the first bit $(p=1, j=0, i=0)$, the correlator output is defined as (Here $\mathrm{M}=\mathrm{N})$ : 


$$
\begin{gathered}
Z_{0,0}=\sum_{k=0}^{M-1} W_{0, k} \sum_{n=0}^{R-1}\left(x_{r(0, n+k R)}+\xi_{(0, n+k R)}\right) \times \\
\left(\frac{1}{M} \sum_{j=0}^{M-1} \sum_{i=0}^{M-1} \sum_{k=0}^{M-1} \sum_{n=0}^{R-1} a_{i+j M} x_{r(j, n+k R)} W_{i k}+\xi_{(n+M R+k R)}\right)
\end{gathered}
$$

Also,

$$
\begin{aligned}
& Z_{0,0}=\sum_{k=0}^{M-1} W_{1, k} \sum_{n=0}^{R-1}\left\{\frac{1}{M} \sum_{j=0}^{M-1} \sum_{i=0}^{M-1} W_{i k} a_{i+j M} x_{r(j, n+k R)} x_{r(0, n+k R)}+\right. \\
& \quad\left(\frac{1}{M} \sum_{j=0}^{M-1} \sum_{i=0}^{M-1} a_{i+j M} x_{r(j, n+k R)} W_{i k} \xi_{(0, n+k R)}\right)+x_{r\left(0, n+k R-\tau_{l}\right)} \xi_{(n+M R+k R)}+ \\
& \left.\xi_{(0, n+k R)} \xi_{(n+M R+k R)}\right\}
\end{aligned}
$$

Use the two expressions; $\sum_{n=0}^{R-1} x_{r(0, n+k R)} x_{r(j, n+k R)} \approx 0$ and $\sum_{n=0}^{R-1} x_{g 0(n)} x_{g(j, n)} \approx 0$, for $j \neq 0$. Also, substitute $a_{i+\mathrm{jM}}=+1$, then (14) becomes

$$
\begin{gathered}
Z_{0,0}=\sum_{k=0}^{M-1} W_{1, k} \sum_{n=0}^{R-1}\left\{\frac{1}{M} W_{i k} x_{r(0, n+k R)}^{2}+\left(\frac{1}{M} \sum_{j=0}^{M-1} \sum_{i=0}^{M-1} x_{r(j, n+k R)} W_{i k} \xi_{(0, n+k R)}\right)+\right. \\
\left.x_{r(0, n+k R)} \xi_{(n+M R+k R)}+\xi_{(0, n+k R)} \xi_{(n+M R+k R)}\right\}
\end{gathered}
$$

The first term in (14) represents the required energy signal while the remaining terms are the interference energy signals. The conditional average and the variance of $\mathrm{Z}_{0,0}$ are found to be respectively as

$$
\begin{aligned}
& E\left[Z_{0,0}\right]=\frac{1}{M} M R E\left[\left(x_{g 0}^{n}\right)^{2}\right] \\
& V\left[Z_{0,0}\right]=\operatorname{MRE} E\left[\left(x_{g 0}^{n}\right)^{2}\right] \frac{N_{0}}{2}+\frac{1}{M^{2}} M^{3} R E\left[\left(x_{g 0}^{n}\right)^{2}\right] \frac{N_{0}}{2}+M R \frac{N_{0}}{2} \frac{N_{0}}{2}
\end{aligned}
$$

where E[.] and V[.] are the average and variance function respectively. Define $E\left[\left(x_{g 0}^{n}\right)^{2}\right]=\frac{M P E_{b}}{(1+P) R}$ in terms energy per bit and substitute it in (16) and (17) to get

$$
\begin{aligned}
& E\left[Z_{0,0}\right]=\frac{P M E_{b}}{(1+P)} \\
& V\left[Z_{0,0}\right]=\frac{2 P M^{2}}{(1+P)} E_{b} \frac{N_{0}}{2}+M R \frac{N_{0}^{2}}{4}
\end{aligned}
$$

Considered $Z_{0,0}$ as Gaussian random variables [4] then The BER theory of the OFDM-OMCS-DCSK can be calculated in terms of the complementary error function (erfc) as

$$
B E R=\frac{1}{2} \operatorname{erfc}\left(\frac{E\left[z_{0,0}\right]}{\sqrt{2 V\left[z_{0,0}\right]}}\right)
$$

Substituting (18) and (19) into (20) and rearranged them to obtain the BER of OFDM-OMCS-DCSK over AWGN as

$$
B E R_{A W G N}=\frac{1}{2} \operatorname{erfc}\left(\frac{2(1+P)}{P} \frac{N_{0}}{E_{b}}+\frac{R(1+P)^{2}}{2 M P^{2}}\left(\frac{N_{0}}{E_{b}}\right)^{2}\right)^{-\frac{1}{2}}
$$

\subsubsection{BER over a multipath Rayleigh channel}

The BER theory for multipath channel can be derived starting from (21) by replacing each $E_{b} / N_{0}$ by $\gamma_{b}=\sum_{l=1}^{L} \alpha_{l}^{2} E_{b} / N_{0}$, where $\alpha_{l}, l=1,2, \ldots, L$ is identically distributed Rayleigh fading gains. We assume here the delay paths, $\tau_{l}, l=1,2, \ldots ., L$, are neglected due to they are maximum value is taken in the range $0<\tau_{\max } \ll(N+M) R$. The BER of OFDM-OMCS-DCSK under multipath fading channel becomes.

$$
B E R_{\text {Multipath }}=\frac{1}{2} \operatorname{erfc}\left(\frac{2(1+P)}{P \gamma_{b}}+\frac{R(1+P)^{2}}{2 M P^{2} \gamma_{b}^{2}}\right)^{-\frac{1}{2}}
$$

Defining $\overline{\gamma_{l}}=E\left[\alpha_{l}^{2}\right] E_{b} / N_{0}$, the probability density function of $\gamma_{b}$ was reported in [31], which is given by 


$$
f\left(\gamma_{b}\right)=\frac{1}{{\overline{\gamma_{l}}}^{L}(L-1) !} e^{\frac{\gamma_{b}}{\bar{\gamma}_{l}}}
$$

Finally, the overall BER expression for OFDM-OMCS-DCSK system over multipath Rayleigh fading channel is calculated by averaging the conditional BER formula in (22) over $\gamma_{b}$, as

$$
B E R_{O F D M-O M C S-D C S K}=\int_{0}^{+\infty} B E R\left(\gamma_{b}\right) \times f\left(\gamma_{b}\right) d \gamma_{b}
$$

\subsection{Bandwidth Efficiency}

In this subsection, the percentage factors of bandwidth efficiency (BE) of OMCS-DCSK, and OFDM- OMCS-DCSK respectively are expressed as at $\mathrm{M}=\mathrm{N}$

$$
\begin{aligned}
& B E_{\text {OMCS-DCSK }}=\frac{M}{2 R} \times 100 \% \\
& B E_{\text {OFDM-OMCS-DCSK }}=\frac{M P}{(1+P) R} \times 100 \%
\end{aligned}
$$

where $B E_{\text {OMCS-DCSK }}$ and $B E_{\text {OFDM-OMCS-DCSK }}$ are the percentage form of BE of OMCS-DCSK and OFDM- OMCS-DCSK systems respectively. Figure 6 shows the percentage form of BE for different values of $\mathrm{M}$ and $\mathrm{P}$. It can be seen that increasing $\mathrm{M}$ or $\mathrm{P}$ will improve $\mathrm{BE}$ of the proposed system compared with the OMCS-DCSK system. For instance, when $\mathrm{R}=100$ and $\mathrm{P}=3, B E_{\text {OFDM-OMCS-DCSK }}=6 \%, 12 \%, 24 \%, 48 \%$ for $\mathrm{M}=8,16,32$ and 64 respectively.

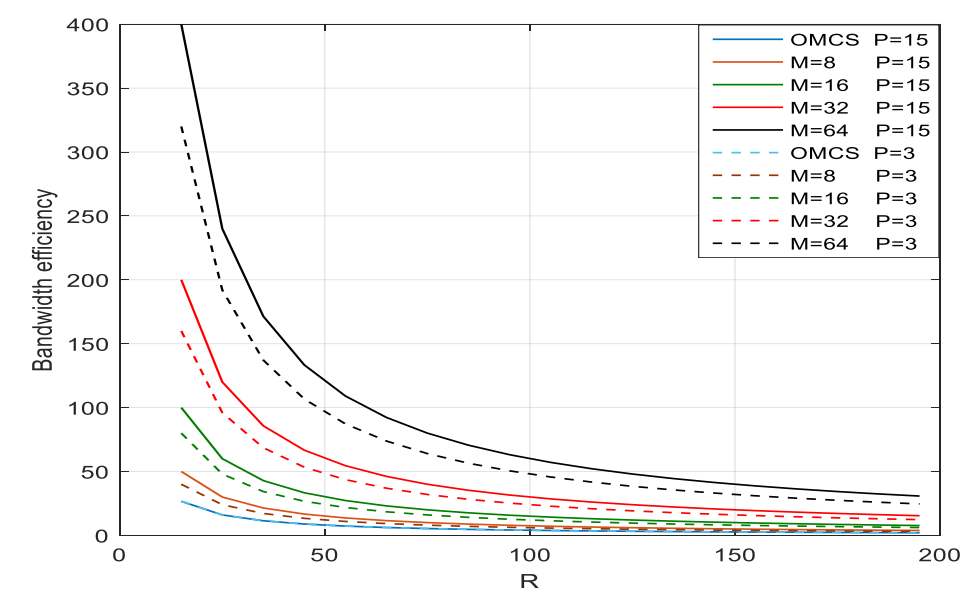

Figure 6. $B E$ comparisons between OMCS-DCSK and the proposed system versus $R$ values and $M=8,16,32$, and64 at $\mathrm{P}=3$ and 15

\section{SIMULATION RESULTS AND DISCUSSIONS}

In this section, the performance of the OFDM-OMCS-DCSK system in BER over AWGN and multipath Rayleigh fading channels is compared with OMCS-DCSK and OFDM-DCSK systems. The parameter settings in this simulation are included: $M=4,8,16$ and 32. Two paths Rayleigh fading channel, $\mathrm{L}=2$, will be used with delays, $\tau_{1}=0$ and $\tau_{2}=2 T_{c}$ and average power gain, $E\left[\alpha_{1}^{2}\right]=2 / 3$ and $E\left[\alpha_{2}^{2}\right]=1 / 3$. Figure 7 shows the BER analytic and simulation curves of OFDM-OMCS-DCSK comparing with OMCS-DCSK over AWGN channel for various values of $\mathrm{M}$ and $\mathrm{P}=255, \mathrm{R}=100$. Figure 8 shows comparison BER performance between OFDM-DCSK, OMCS-DCSK and the proposed system under AWGN channel with the same $\mathrm{R}=\beta, \mathrm{M}=\mathrm{N}=32$ and $\mathrm{P}=31$. From these figures, it can be seen that the BER analytic is matching with the simulation results. The BER performance enhances with increasing the value of $\mathrm{M}$ and $\mathrm{N}$ and outperforms the BER performance of OMCS-DCSK and OFDM-DCSK. Besides, the OFDM-OMCS-DCSK system is carried 31744 bits in each frame while OMCS-DCSK and OFDM-DCSK carry 1024 and 31 bits respectively. It can be observed that over AWGN at BER $=10^{-3}$, the SNR gain of the proposed system compared to OFDM-DCSK and MCS-DCSK are 1.1 and $2.85 \mathrm{~dB}$ respectively. 


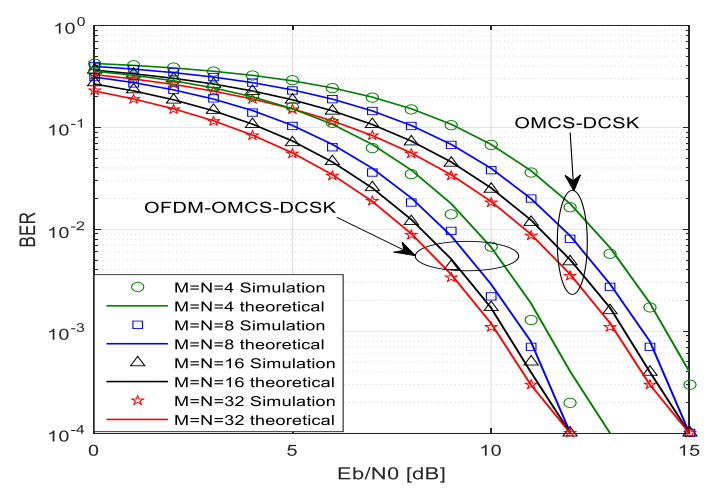

Figure 7. BER comparison OFDM-OMCS-DCSK and OMCS-DCSK systems over AWGN channel with $R=100, P=255$ and different values of $M$

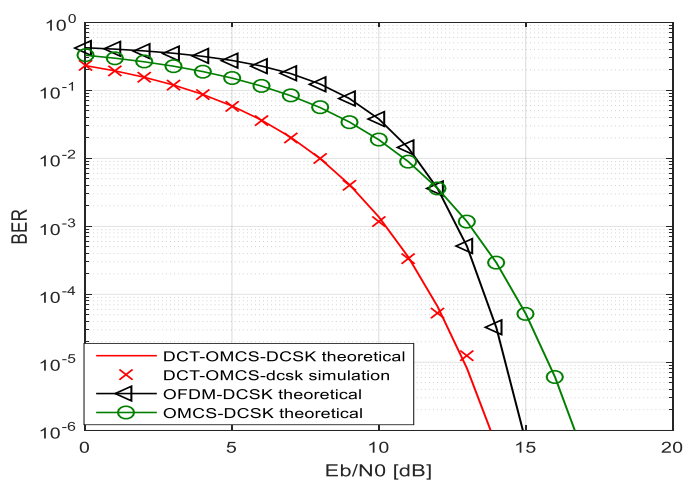

Figure 8. BER comparison between BER OFDMOMCS-DCSK, OMCS-DCSK and OFDM-DCSK systems over AWGN channel with $R=100, P=31$ and $M=32$

Figure 9 shows BER comparison OFDM-OMCS-DCSK, OFDM-DCSK and OMCS-DCSK under two path Rayleigh fading channel at $P=31, \mathrm{R}=100$ and different values of $\mathrm{M}$. In addition, that compared at the same channels with same $P=31, \mathrm{R}=\beta=100$ and $\mathrm{M}=\mathrm{N}=8$ proposal. It can be observed the BER performance enhanced with increasing the value of M. Besides, the OFDM-OMCS-DCSK system is carried 1984 bits in each frame while OMCS-DCSK and OFDM-DCSK carry 64 and 31bits respectively. Also, the SNR gain the OFDM-OMCS-DCSK system at BER $=10^{-3}$ comparing with OFDM-DCSK and OMCS-DCSK are 4, $4.2 \mathrm{~dB}$ respectively. Therefore, the OFDM-OMCS-DCSK system has excellent performance and highest capability of transmitted bits comparing with OFDM-DCSK and OMCS-DCSK systems.

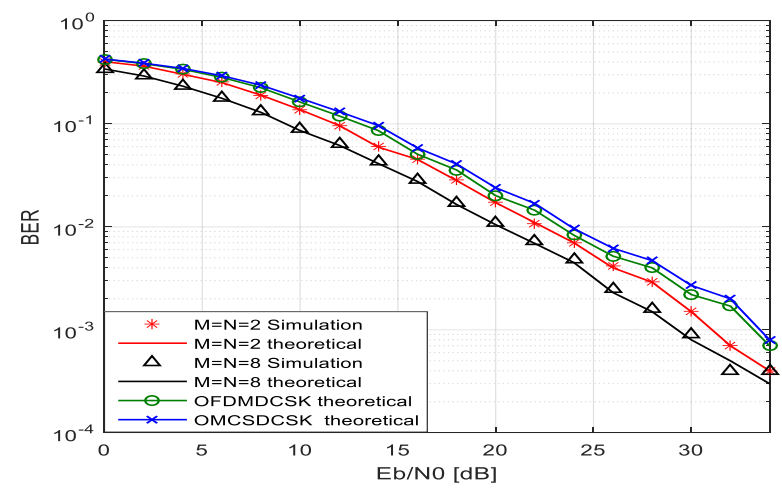

Figure 9. BER Comparison between OFDM-OMCS-DCSK, OMCS-DCSK and OFDM-DCSK systems over two paths Rayleigh fading channel with $\beta=R=100, P=31$ and $M=N=2$ and 8

\section{CONCLUSION}

In this paper, a new high data rate version of non-coherent chaotic communication is proposed, that is combined the OMCS-DCSK system with the OFDM system. The proposed system utilizes the Gram-Schmidt algorithm in the OCVSK system and the Walsh functions in the MCS-DCSK system to generate huge orthogonal sets that is capable to carry high data rate with efficient way. The OFDM-OMCS-DCSK system can transmit MNP bits, where $\mathrm{N}$ is orthogonal chaotic vectors, $\mathrm{M}$ is Walsh functions and $\mathrm{P}$ is the number of subcarriers in the same frame. We need to transmit the $N$ chaotic reference signals to detect the transmitted bits differentially. Also, the analytic expression of BER at AWGN channel and multipath fading channels are derived and compared with BER simulation at different parameters. Besides, the OFDM-OMCS-DCSK is compared with OMCS-DCSK and OFDM-DCSK systems and the results show that the proposed system can improve the bandwidth efficiency comparing with OMCS-DCSK and OFDM- DCSK systems and has the best BER performance. 


\section{ACKNOWLEDGEMENTS}

This work is supported by the college of Engineering/Mustansiriyah University (https://webmail.uomustansiriyah.edu.iq).

\section{REFERENCES}

[1] Kurian A. P., et al., "Performance enhancement of DS-CDMA system using chaotic complex spreading sequence," IEEE Transaction Wireless Communication, vol. 4, no. 3, pp. 984-989, 2005.

[2] Lau F. C. M. and Tse C. K., "Chaos-based digital communication systems," New York, Springer-Verlag, 2003.

[3] Kaddoum G., "Wireless chaos-based communication systems: a comprehensive survey," IEEE Access, vol. 4, pp. 2621-2648, 2016.

[4] Xia Y., et al., "Performance of differential chaos-shift-keying digital communication systems over a multipath fading channel with delay spread," IEEE Transactions on Circuits and System, vol. 51, no. 12, pp. 680-684, 2004.

[5] Yu J. and Yao Y. D.,"Detection performance of chaotic spreading LPI waveforms," IEEE Transaction on Wireless Communication, vol. 4, no. 2, pp. 390-396, 2005.

[6] H. A. Abdullah and H. N. Abdullah, "FPGA implementation of color image encryption using a new chaotic map," IJEECS, vol. 13, no. 1, pp. 129-137, 2019.

[7] Fadhel S., et al., "Chaos Image Encryption Methods: A Survey Study," Bulletin of Electrical Engineering and Informatics (BEEI), vol. 6, no. 1, pp. 99-104, 2017.

[8] G. Kolumban, et al., "Differential chaos shift keying: A robust coding for chaoscommunication," in Proc. NDES96, pp. 87-92, 1996.

[9] F. S. Hasan and A. A. Valenzuela, "Design and Analysis of an OFDM-based orthogonal chaotic vector shift keying communication system," IEEE Access, vol. 6, pp. 46322-46333, 2018.

[10] Z. Galias and G. M. Maggio, "Quadrature chaos-shift keying: Theory and performance analysis," IEEE Trans. Circuits Syst.-I, vol. 48, no. 12, pp. 1510-1519, 2001.

[11] L. Wang, et al., "Design and performance analysis of a new multiresolution M-ary differential chaos shift keying communication system," IEEE Trans. Wireless Commun., vol. 14, no. 9, pp. 5197-5208, 2015.

[12] S. Wang and X. Wang, "M-DCSK-based chaotic communications in MIMO multipath channels with no channel state information," IEEE Trans. Circuits Syst. II, Exp. Briefs, vol. 57, no. 12, pp. 1001-1005, 2010.

[13] T. J. Wren T. C. Yang, "Orthogonal chaotic vector shift keying in digital communications," IET Communications, vol. 4, no. 6, pp. 739-753, 2010.

[14] W. K. Xu and L. Wang, "A novel differential chaos shift keying modulation scheme," Int. J. Bifurcation Chaos, vol. 21, no. 3, pp. 799-814, 2011.

[15] H. Yang and G. P. Jiang, "High-efficiency differential-chaos-shift-keying scheme for chaos-based noncoherent communication," IEEE Transactions on Circuits and Systems II: Express Briefs, vol. 59, no. 5, pp. 312-316, 2012.

[16] Kaddoum G., et al., "Design of a secure multi-carrier DCSK system," IEEE International symposium on wireless communication systems, pp. 964-8, 2012.

[17] Kaddoum G., and Gagnon F., "Design of a high-data-rate differential chaos-shift keying system," IEEE Transactions on Circuits and Systems II: Express Briefs, vol. 59, no. 7, pp. 448-452, 2012.

[18] H. Yang and G. P. Jiang, "Reference-modulated DCSK: A novel chaotic communication scheme," IEEE Transactions on Circuits and Systems II: Express Briefs, vol. 60, no. 4, pp. 232-236, 2013.

[19] Huang T., et al., "Multilevel code-shifted differential-chaos-shift-keying system," IET Communications, vol. 10, no. 10, pp. 1189-1195, 2016.

[20] H. Yang, et al., "System design and performance analysis of orthogonal multi-level differential chaos shift keying modulation scheme," IEEE Trans. Circuits Syst.-I, vol. 63, no. 1, pp. 146-156, 2016.

[21] H. Yang, et al., "Phase-separated DCSK: A simple delay-component- free solution for chaotic communications," IEEE Transactions on Circuits and Systems II: Express Briefs, vol. 61, no. 12, pp. 967-971, 2014.

[22] Kaddoum G., et al., "I-DCSK: An improved non-coherent communication system architecture," IEEE Transactions on Circuits and Systems II: Express Briefs, vol. 62, no. 9, pp. 901-905, 2015.

[23] G. Kaddoum, et al., "Design of a short reference non-coherent chaos-based communication systems," IEEE Transactions on Communications, vol. 64, no. 2, pp. 680-689, 2016.

[24] M. Herceg, et al., "Commutation code index DCSK modulation technique for high-data-ratecommunication systems," IEEE Transactions on Circuits and Systems, 2018.

[25] P. Manhas and M. K. Soni, "Comparison of OFDM System in terms of BER using Different Transform and Channel Coding," Indonesian Journal of Electrical Engineering and Computer Science, vol. 1, no. 1, pp. 153-158, 2016.

[26] Li S., et al., "Design and analysis of an OFDM-based differential chaos shift keying communication system," Journal of Communications, vol. 10, no. 3, pp. 199-205, 2015.

[27] F. S. Hasan, "Design and analysis of an OFDM-based short reference quadrature chaos shift keying communication system," Wireless Personal Communications, vol. 96, no. 2, pp. 2205-2222, 2017.

[28] Kaddoum G., et al., "Design of a secure multi-carrier DCSK system," IEEE International symposium on wireless communication systems, pp. 964-968, 2012.

[29] Kaddoum G., et al., "Design and analysis of a multi-carrier differential chaos shift keying communication system," IEEE Transactions on Communications, vol. 61, no. 8, pp. 3281-3291, 2013. 
[30] G. Kaddoum, et al., "Code index modulation:A high data rate and energy efficient communication system," IEEE Commun Lett, vol. 19, no. 2, pp. 175-178, 2015.

[31] G. Kaddoum, et al., "Generalized code index modulation technique for high-data-rate communication systems," IEEE Trans. Veh. Technol, vol. 65, no. 9, pp. 7000-7009, 2016.

[32] G. Cheng, et al., "Carrier index differential chaos shift keying modulation," IEEE Trans. Circuits Syst. II, Exp. Briefs, 2016.

[33] W. Xu, et al., "Code-shifted differential chaos shif keying with code index modulation for high data rate transmission," IEEE Trans. Commun., 2017.

[34] X. Cai, et al., "An M-ary Orthogonal Multilevel Differential Chaos Shift Keying System with Code Index Modulation,” IEEE Trans. Commun., vol. 67 , no. 7, pp. 4835-4847, 2019.

\section{BIOGRAPHIES OF AUTHORS}

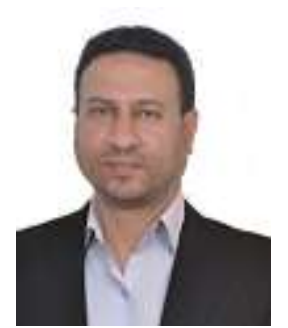

Fadhil S. Hasan was born in Baghdad, Iraq in 1978. He received his B.Sc. degree in Electrical Engineering in 2000 and his M.Sc. degree in Electronics and Communication Engineering in 2003, both from the Mustansiriyah University, Iraq. He received Ph.D. degree in 2013 in Electronics and Communication Engineering from the Basrah University, Iraq. In 2005, he joined the faculty of Engineering at the Mustansiriyah University in Baghdad. His recent research activities are Wireless Communication Systems, Multicarrier System, Wavelet based OFDM, MIMO System, Speech Signal Processing, Chaotic Modulation, FPGA and Xilinx System Generator based Communication System. Now he has been an Assist. Prof. at the Mustansiriyah University, Iraq.

Email: fadel_sahib@uomustansiriyah.edu.iq

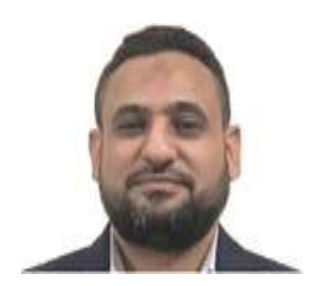

Hayder F. Fahad was born in Baghdad, Iraq in 1982. He received his B.Sc. degree in Electrical Engineering in 2011 from the faculty of engineering at the Mustansiriyah University in Baghdad, Iraq.

Email: haider.fadhil.fahad@gmail.com 